\title{
Dealing with Uncertainties in Manufacturing Process Simulations
}

\author{
Patrick Ruediger ${ }^{1, a *}$, Hans Hagen ${ }^{2, b}$ \\ ${ }^{1}$ TU Kaiserlautern, Dep. of Computer Science, Germany \\ ${ }^{2}$ TU Kaiserslautern, Dep. of Computer Science, Germany \\ aruediger@rhrk.uni-kl.de, bhagen@cs.uni-kl.de
}

Keywords: process uncertainty, manufacturing process, assembly process simulation, human factors, simulation validation, effects of uncertainty, simulation

\begin{abstract}
Uncertainty in manufacturing processes is as old as the manufacturing process itself. Simulations on the other hand are always certain in their outcome based on the chosen parameters. Nevertheless it makes sense to incorporate uncertainties in the simulation for validation and analysis of the real and simulated processes. This paper aims on highlighting the importance of an accurate understanding and measurement of uncertainty for simulation validation and thus to increase the significance and acceptance of simulation results in the working environment.
\end{abstract}

\section{Introduction}

Simulations play a crucial role in nowadays manufacturing systems [13] and are used through all phases of manufacturing. Starting at the planning and design phase to test new prototypes and all the way up to simulate the real time production parameters. The benefit of simulations is clear nowadays [3], but it is not always clear why something should be simulated [16]. The goal of simulations therefor is often misunderstood and simulations are far to often seen as a replacement of physical processes, which leads to a low acceptance of simulation development and results through out the company. Instead the aim of simulations should be the analysis of processes with the necessary flexibility for upcoming changes. Generalization therefor is the key factor of a simulation, but the significance is a limiting factor. In order to have significant results the simulation should match real achievable situations in the process. Typically those two factors contradict each other at a given point. It makes sense to formulate it as an optimization problem for simulation development:

$$
\begin{gathered}
\text { Generalization }:=\{\text { situations, that can be simulated }\} \\
\text { max } \mid \text { Generalization } \mid, \text { with significance }>s_{0}
\end{gathered}
$$

The generalization can be easily described as a set of simulate-able situations. Maximizing the number of those will maximize the generalization. Of course a more complex description of generalization could be used as well, but the focus in this paper is on the second aspect of the optimization problem, the significance. The lack of a unitary understanding of significance in this context makes it even harder to find a proper threshold for it. Therefor we propose to replace it with the more intended meaning certainty and assume that the semantic correlation between certainty and significance is given in the scope of manufacturing processes. Even one has to admit, that there is no study yet proving this assumption scientifically, our empirical experiences with industry partners has shown that this is true and the that the most differences in the meaning are cause due to language barriers. It figures out that using uncertainty instead of certainty suits better in most cases and is nearer to the meaning of error in statistics, which is a good analogous for uncertainty in simulation validation with real process data. The optimization problem then changes to:

$$
\max \mid \text { Generalization } \mid, \text { with uncertainty }<\mu_{0}
$$


Now that we have formulated our optimization problem, it can be checked in each step of the validation process, if an improvement was made in context to the overall process uncertainty. Nonetheless it remains a challenge to communicate the results with respect to the present uncertainties. Therefor we first take a closer look at the difference between precision and uncertainty and how to measure those. Then the three dimensions of simulation validation are introduced to situate uncertainty in the validation process. Last we talk about how to communicate and incorporate uncertainty in the whole process and finish with our final conclusions.

Related work Zouaoui et.al. [26],[27] and Barton et. al. [1] are talking in detail on how to deal with uncertainties in the input model. Where Zou et. al. [25] and McKay et.al. [11] are investigating the problem of analyzing and evaluating uncertainty. Du and Chen [7] and Hills [9] provide models on how to manage uncertainty in the process and therefor also provide useful ideas on how to analyze uncertainty. A very advanced approach for its time was proposed by Su and Lee [17], where they analyzed the uncertainty of a process by controlled manipulation of uncertainty factors. A good resource and starting point for visualizing uncertainties is made by Potter et. al. [15].

\section{Precision vs Uncertainty}

Now that the optimization problem is stated, the question arises how to check if the conditions hold. As we are mainly collaborating with engineers from the metrology and quality assurance department, it stands to reason using the definitions of precision and uncertainty from the 2007 International vocabulary of metrology [5]. As long as we mainly investigate the uncertainty of input parameters these definitions are sufficient enough. If we also want to take into account model uncertainties the Committee on Mathematical Foundations of Verification, Validation, and Uncertainty Quantification already published a profound study with extensive examples [4].

Definition 1. Measurement uncertainty is a non-negative parameter characterizing the dispersion of the quantity values being attributed to a measurand, based on the information used

"NOTE 1 Measurement uncertainty includes components arising from systematic effects, such as components associated with corrections and the assigned quantity values of measurement standards, as well as the definitional uncertainty. Sometimes estimated systematic effects are not corrected for but, instead, associated measurement uncertainty components are incorporated.

NOTE 2 The parameter may be, for example, a standard deviation called standard measurement uncertainty (or a specified multiple of it), or the half-width of an interval, having a stated coverage probability.

NOTE 3 Measurement uncertainty comprises, in general, many components. Some of these may be evaluated by Type A evaluation of measurement uncertainty from the statistical distribution of the quantity values from series of measurements and can be characterized by standard deviations. The other components, which may be evaluated by Type B evaluation of measurement uncertainty, can also be characterized by standard deviations, evaluated from probability density functions based on experience or other information.

NOTE 4 In general, for a given set of information, it is understood that the measurement uncertainty is associated with a stated quantity value attributed to the measurand. A modification of this value results in a modification of the associated uncertainty." from The 2007 International Vocabulary of Metrology Point 2.26 [5]

Definition 2. Measurement precision is the closeness of agreement between indications or measured quantity values obtained by replicate measurements on the same or similar objects under specified conditions. 
"NOTE 1 Measurement precision is usually expressed numerically by measures of imprecision, such as standard deviation, variance, or coefficient of variation under the specified conditions of measurement.

NOTE 2 The 'specified conditions' can be, for example, repeatability conditions of measurement, intermediate precision conditions of measurement, or reproducibility conditions of measurement (see ISO 5725- 3:1994).

NOTE 3 Measurement precision is used to define measurement repeatability, intermediate measurement precision, and measurement reproducibility.

NOTE 4 Sometimes "measurement precision" is erroneously used to mean measurement accuracy." from The 2007 International Vocabulary of Metrology Point 2.15 [5]

Precision and uncertainty are picked because they represent the two extrema in case of their practical determinability. Where precision is clearly defined and quantified, but also very limited to a special subset of the process. Uncertainty on the other hand is more general but lacks of a clear definition and quantification. Still uncertainty is preferable when talking about complex processes, when not only syntactical and statistical information matters, but also semantical and even more important the embedding context. Only this way, real significant statements can be made. An example here for is easily found.

Assuming we want to build a ship. The main goal should be, that the ship is tight. So if we built up the hull of our ship with multiple parts, simulating the assembly process is a crucial factor for the ships tightness. Now assume that we want to validate our simulation result with a real assembled hull that was measured with a high precision optical laser. The laser has a resolution of approximately $10-100 \mathrm{~nm}$ and the ship is tight when the gab between the parts is smaller than $1 e^{6} \mathrm{~nm}=1 \mathrm{~mm}$. So clearly the precision of the measurement is high enough for the validation. The simulation mesh has a resolution of $\frac{1}{2} \times 1 \mathrm{~mm}$ (tightness), to match the precision needed for the tightness analysis. If we now just compare our simulation result with the measurement regarding precision, we have to compare multiple measurements with our result and then are able to calculate the overall and median difference and thus determine the precision.

The question arises how much this tells us about the significance and uncertainty of the simulation? Even if we take all differences into account and plot them in an appropriate way, we are still only interested in the tightness of our assembly. So without taking into account the context of our results, like how certain is the real process, how well is the process defined and if all the influences are understood, it is hard to validate how certain the simulation result is and how good it finally fits for the tightness analysis in the assembly process. This is way uncertainty has to be emphasized and defined in the whole process.

It makes sense now to built up the quantification of uncertainty out of the division of the process in measurable subprocesses and their connection and correlation in addition to soft characteristics like human influence factors, which are usually hard to measure precisely. Taylor et. al. [20] gives a broad overview and proposes a general theory of how to approach such a quantification problem.

\section{The 3-dimensions of simulation validation}

In order to get a better understanding for the communication problem behind uncertainty and simulation validation, a closer look at the dimensions of validation is beneficial. Therefor we classified the validation in three dimensions.

- Effort

- Completeness

- Certainty 


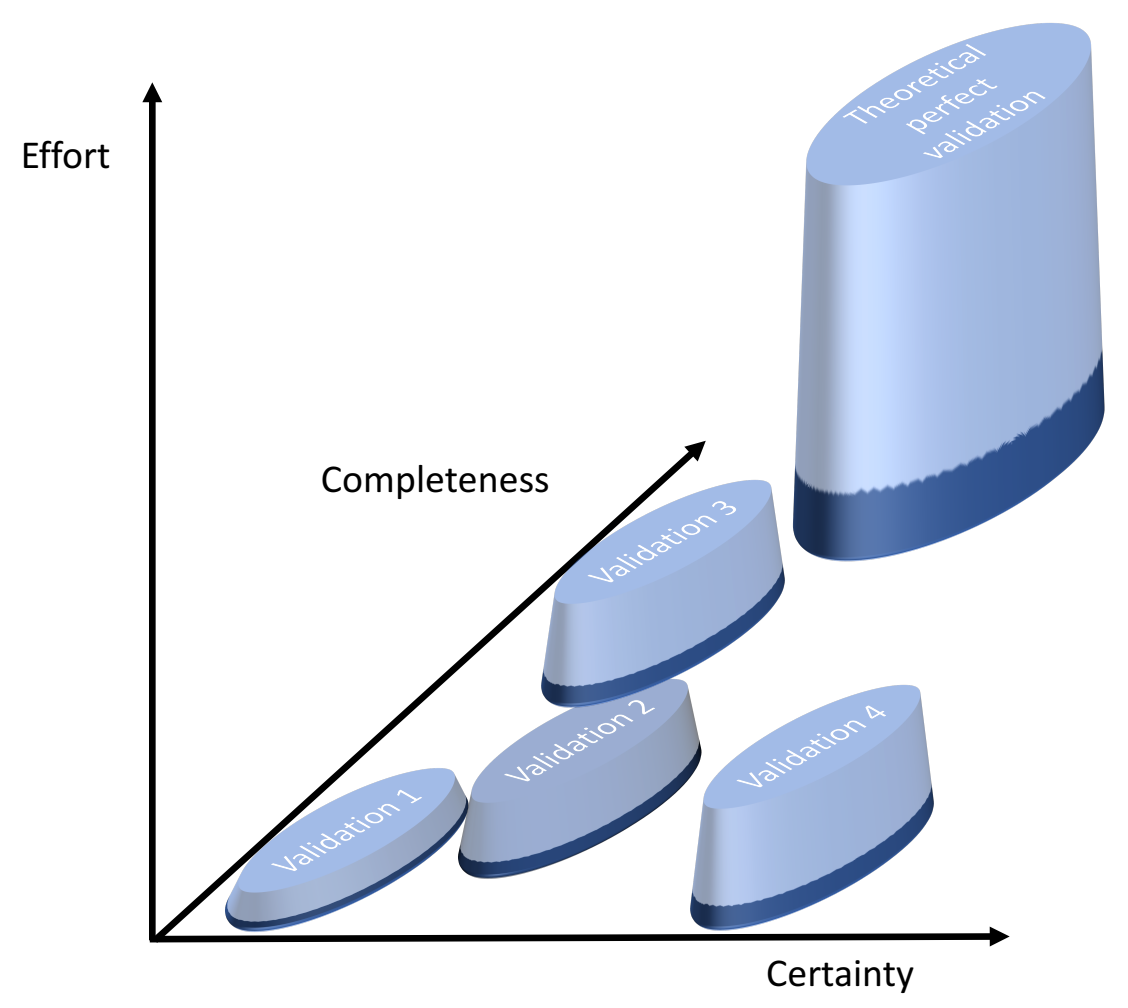

Fig. 1: Depiction of the three dimensions of simulation validation. Each new phase of validation is always a trade-off between effort, completeness and certainty. The theoretical perfect validation is usually not achievable as the knowledge about the simulated process is never complete, nor certain and the effort is no longer in any rational relationship to it.

Validation completeness is the measurement of how detailed a the validation is supposed to be. This takes into account that for a full coverage of completeness in validation one has to know every possible mechanism and its effects of a process. Obviously a complete coverage can only be achieved in theory and depends on the completeness of the process embedding context. Usually the maximum completeness is determined by the actual knowledge and understanding of the underlying processes. As one goal of simulations is to enhance exactly this knowledge, even a realistic completeness could not be achieved. However sometimes completeness could also not be achieved because of the lack of proper measurement methods for certain effects or because the measurement itself is incomplete or uncertain. Human factors for example are always crucial in this context, as they are both incomplete in their measurable description and uncertain regarding their behavior. The lack of completeness therefor always correlates with the achievable certainty of a validation.

Validation certainty is the measurement of how certain the validation results are (for an easier graphical depiction the positive formulation is used here). This takes into account the cumulative precision of the used measurement results and the effect of how well the input parameters could be determined. The latter is the most critical one, as it is also affected by how well the overall process is understood and how well material parameters or other derived parameters are suited for the simulated process. In most cases there are no special parameters derived and one has to deal with experimentally determined ones, which makes it hard to quantify the suitability or correctness of the parameters. Far too often the simulation itself is used to determine the suitability of a certain parameter, not taking into account that the parameter could have side effects and correlations with the other parameters.

In industry the necessary effort for the validation should not be forgotten. Validation could be a very time-consuming and costly task and therefor the methods should also emphasize the given resources to achieve the best trade-off between certainty and completeness. 


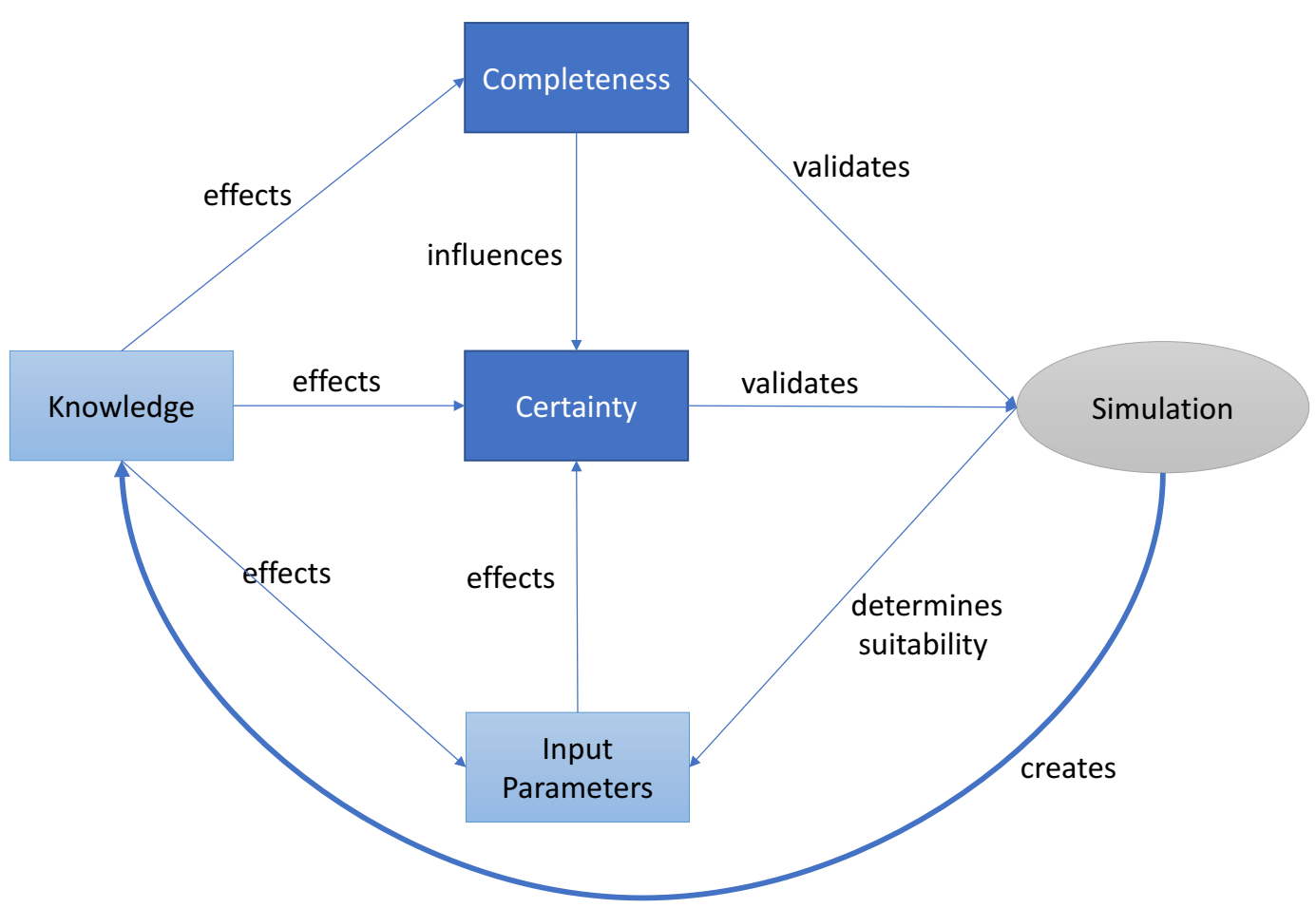

Fig. 2: The curse of dimensions for simulation validation. Since everything is connected it is hard to draw a clear line at which state the validation result holds. Incorporating the existing knowledge is a key factor for a successful and significant validation of the simulation, even if there is a loop in the validation process.

This way we can build up the dimensions of our validation systems to allow for a classification of methods to choose from. As one can see from Fig. 2 that a good result is hardly achievable with only one validation. To overcome the effects of mutual manipulation a series of validations is necessary with a clear documentation of the used dependencies.

In order to decrease the uncertainty there are three main steps in each validation phase. First one needs to isolate the effects as much as possible to overcome the curse of dimensionality regarding the dimensions of possible influences and effects from knowledge, completeness and input parameters (see Fig.2). It seems much easier to correlate and compare two effects than 5 or more at the same time. Thus Divide \& Conquer is the strategy to go for. But one has to keep in mind, that it is not always possible nor feasible for each effect and influence to divide it to the lowest level. Second the correlation and dependencies between effects need to be determined such that one can perform the "conquer" step. The conquering step is also not free of complications as there is no guarantee for every division that the integration can be made without the loss of information and thus induce more uncertainty instead of lowering it. The third step is to distinguish between systematic and unsystematic effects. In the report of the national research council ([4] p.14 Fig 1.3) they also discussed this problematic in detail based on the weapon failure example. The last step is of particular importance as a simulation is usually only capable of mapping the systematic effects with a quantified certainty. Systematic effects are those that can be reproduced, scientifically explained and do have a precise and formalized description. All other effects are considered unsystematic.

In particular all effects where humans are involved should be handled carefully in this context. There are two main factors for which we distinguish the human as unsystematic or systematic in manufacturing processes. The one is the level of detail or precision range. High requirements on the precision lead to a more unpredictable behavior of the human worker when the precision range approaches the 
human motoric capabilities thus classifying it as unsystematic. The second one is the degree of variability in the modeled assembling process. Typically humans are not deterministic in their behavior when having multiple options that are valid. The human flexibility is always a curse and a blessing in assembling processes, but when it comes to simulation the question is always how to incorporate the human. Even it is mostly an unsystematic effect on the uncertainty of the process, when there are multiple options available, it can be barely eliminated from the term, as its influence is too high. One way that has been fruitful lately is to allow uncertainties in the input parameters to allow the modeling of human uncertainties in the real process. In assembly processes this is typically achieved by a model describing the play of the parts that can be used. In more general terms, soft boundary conditions can be used in the simulation to incorporate uncertainties in general. Allowing a broader range of possible solutions.

\section{Conclusion}

It has been shown how uncertainty in process simulations can be dealt with. The importance of understanding and analyzing the uncertainty is an old topic in manufacturing processes. With the extended use of simulations and increasing precision and amount of measurement data, this old topic can be analyzed in new ways. Simulations allow for a more clearer and controllable understanding of the underlying effects of a manufacturing process. Emphasizing uncertainty and communicating it to all stakeholders further increases the acceptance of the process and the simulation as a beneficial tool. Even further it highlights in a smart way the benefits of using simulations as a tool to gain knowledge about existing processes instead of just replacing prototyping or even existing testing processes. Uncertainty is a powerful aspect of simulations and emphasizing it, directly increases the significance of a simulation. In the future the communication of uncertainties through simulation is getting a major challenge for simulation designs as it further increases the requirements. The trade-off between completeness and a low uncertainty in simulation therefor will still persist.

\section{References}

[1] R. R. Barton, B. L. Nelson, and W. Xie. Quantifying input uncertainty via simulation confidence intervals. INFORMS journal on computing, 26(1):74-87, 2013.

[2] J. W. M. Bertrand and D. R. Muntslag. Production control in engineer-to-order firms. International Journal of Production Economics, 30:3-22, 1993.

[3] D. E. Bray and R. K. Stanley. Nondestructive evaluation: a tool in design, manufacturing and service. CRC press, 1996.

[4] N. R. Council. Assessing the reliability of complex models: mathematical and statistical foundations of verification, validation, and uncertainty quantification. National Academies Press, 2012.

[5] P. De Bièvre. The 2007 International Vocabulary of Metrology (VIM), JCGM 200: 2008 [ISO/IEC Guide 99]: Meeting the need for intercontinentally understood concepts and their associated intercontinentally agreed terms. Clinical biochemistry, 42(4):246-248, 2009.

[6] R. Desai. On fine motion in mechanical assembly in presence of uncertainty. $\mathrm{PhD}$ thesis, $\mathrm{Ph}$. D. thesis, Department of Mechanical Engineering, the Univ. of Michigan, 1989.

[7] X. Du and W. Chen. Methodology for managing the effect of uncertainty in simulation-based design. AIAA journal, 38(8):1471-1478, 2000. 
[8] M. L. Fisher and C. D. Ittner. The impact of product variety on automobile assembly operations: Empirical evidence and simulation analysis. Management science, 45(6):771-786, 1999.

[9] R. G. Hills. Model validation: model parameter and measurement uncertainty. Journal of Heat Transfer, 128(4):339-351, 2006.

[10] P. Jonsson and M. Lesshammar. Evaluation and improvement of manufacturing performance measurement systems-the role of OEE. International Journal of Operations \& Production Management, 19(1):55-78, 1999.

[11] M. D. McKay, J. D. Morrison, and S. C. Upton. Evaluating prediction uncertainty in simulation models. Computer Physics Communications, 117(1-2):44-51, 1999.

[12] T. S. Mujber, T. Szecsi, and M. S. Hashmi. Virtual reality applications in manufacturing process simulation. Journal of materials processing technology, 155:1834-1838, 2004.

[13] G. Ngaile and T. Altan. Simulations of manufacturing processes: Past, present, and future. Advanced technology of plasticity 2002-Proceedings of the 7th ICTP, 1:271-282, 2002.

[14] A. V. Oppenheim, A. S. Willsky, and S. H. Nawab. Signals and systems 2nd ed. New Jersey: Prentice Hall, 1997.

[15] K. Potter, P. Rosen, and C. R. Johnson. From quantification to visualization: A taxonomy of uncertainty visualization approaches. In Uncertainty Quantification in Scientific Computing, pages 226-249. Springer, 2012.

[16] N. F. Roozenburg and J. Eekels. Product design: fundamentals and methods, volume 2. Wiley Chichester, 1995.

[17] S.-F. Su and C. G. Lee. Manipulation and propagation of uncertainty and verification of applicability of actions in assembly tasks. IEEE Transactions on Systems, Man, and Cybernetics, 22(6):1376-1389, 1992.

[18] W. H. Tang and A. Ang. Probability Concepts in Engineering: Emphasis on Applications to Civil \& Environmental Engineering. Wiley, 2007.

[19] Z. Tang, F. J. Liu, S. H. Guo, J. Chang, and J. J. Zhang. Evaluation of coupled finite element/meshfree method for a robust full-scale crashworthiness simulation of railway vehicles. Advances in Mechanical Engineering, 8(4):1687814016642954, 2016.

[20] J. R. Taylor. An Introduction to Error Analysis: The Study of Uncertainties in Physical Measurements. University Science Books, Jan. 1997. Google-Books-ID: giFQcZub80oC.

[21] J. Wang, C. Wang, T. Zhang, and B. Zhong. Comparison of Different Independent Component Analysis Algorithms for Output-Only Modal Analysis. Shock and Vibration, 2016, 2016.

[22] M. Wazed, S. Ahmed, Y. Nukman, and others. Uncertainty factors in real manufacturing environment. 2009.

[23] R. V. Wilson. Estimating uncertainty in computations of two-dimensional separated flows. 1994.

[24] Q. Yu, H. Zhou, and L. Wang. Finite element analysis of relationship between tightening torque and initial load of bolted connections. Advances in Mechanical Engineering, 7(5):1687814015588477, 2015. 
[25] T. Zou, M. Cai, R. Du, and J. Liu. Analyzing the uncertainty of simulation results in accident reconstruction with Response Surface Methodology. Forensic science international, 216(1):49$60,2012$.

[26] F. Zouaoui and J. R. Wilson. Accounting for input model and parameter uncertainty in simulation. In Proceedings of the 33nd conference on Winter simulation, pages 290-299. IEEE Computer Society, 2001.

[27] F. Zouaoui and J. R. Wilson. Accounting for parameter uncertainty in simulation input modeling. Iie Transactions, 35(9):781-792, 2003. 